\title{
The history of cool
}

\section{A look back at pioneering studies of quantum effects at low temperatures.}

\section{Cold Wars: A History of Superconductivity \\ by Jean Matricon \& Georges Waysand Rutgers University Press: 2003. 304 pp. \$65 \\ Philip W. Anderson}

This book, translated from the French, is a history of the field of cryogenics and of the two specifically quantum forms of matter in bulk - superconductivity and superfluidity (although only the first is mentioned in the subtitle). The authors clearly express their enthusiasm (which I share) for the field and its history: the fascination and mystery that it held for the icons of the quantum revolution, from Albert Einstein through Werner Heisenberg to Richard Feynman; the slow and uneven advance of science as it was impeded by personality conflicts, the savagery of the Stalinist regime, and the politics of the real Cold War; the clashes of conflicting ways of doing research in disparate fields; and even, most recently, a case of the deliberate manufacture of scientific results on an unprecedented scale. There is a lot of rich material here that has been, on the whole, neglected.

This history has more than its share of fascinating, larger-than-life figures. It begins with the Dutch entrepreneur Heike Kamerlingh Onnes, who discovered superconductivity. In order to liquefy helium, he created in Leiden the Cryogenic Laboratory, the first industrial-scalelab for pure scientific research and the true precursor of today's CERN, Fermilab and Kamiokande.

Then there were the Russians: Pyotr Kapitsa, Ernest Rutherford's favourite, who was kidnapped by Stalin from Cambridge, UK, to carry on his helium research in Russia; Lev Shubnikov, who founded the great Kharkov lab but was murdered by Stalin in 1938 , some 15 years before his wonderful experiments demonstrating 'type II' superconductivity in alloys - the phase that makes possible high-field magnets such as those used in magnetic resonance imaging (MRI) — were understood; and the brilliant figure of Lev Landau, Russia's greatest theorist.

We also meet Fritz London, the underrecognized genius who was denied recognition for his great contribution to superfluidity - the realization that it was a Bose-Einstein condensate - by Landau. There is John Bardeen, who, with Leon Cooper and Robert Schrieffer, proposed the BCS theory of superconductivity, but only after first winning a Nobel prize for the transistor. And finally we come across the charismatic Bernd Matthias, the 'alchemist' guru of what the authors call the "age of materials". Matthias

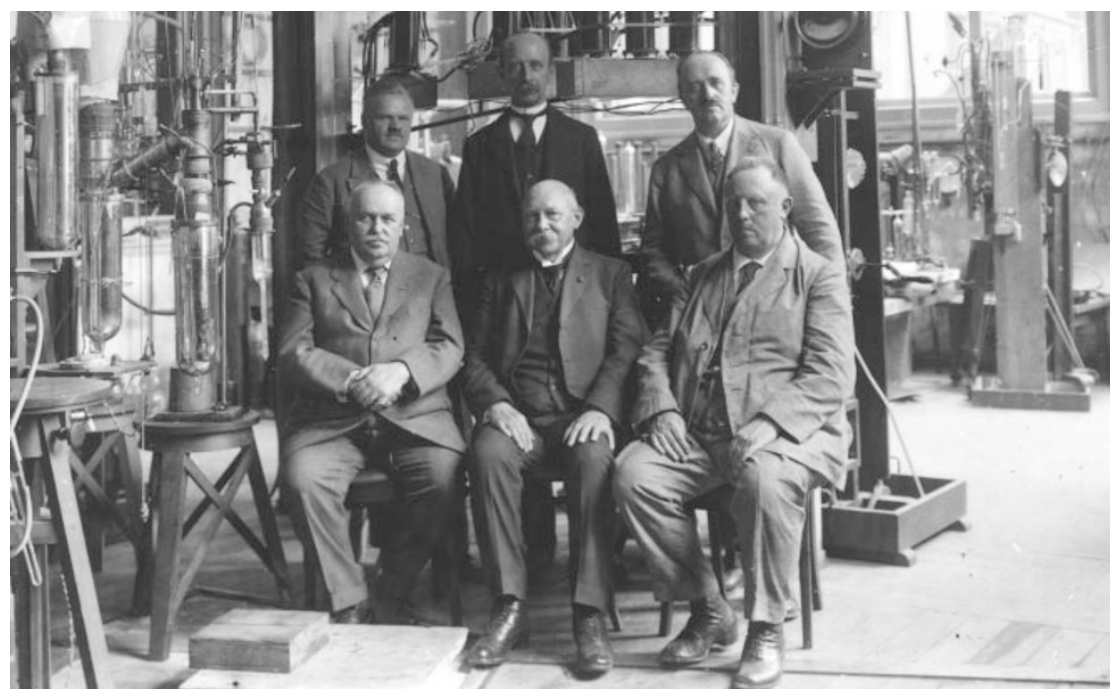

Super stars? Researchers at Heike Kamerlingh Onnes' lab in Leiden discovered superconductivity.

died too early to catch the breakthroughs in high-temperature superconductivity made by his followers and students.

Having said all that, throughout the book I kept encountering historical blunders and misapprehensions that left me wondering how sound the rest of it was. For instance, in the space of two pages there are three dubious historical judgements. To begin with, "The history of physics was indelibly marked by WWII, primarily because of the ... Bomb," write the authors, but more physicists worked on radar than on the bomb, and much more technology resulted from it. "Los Alamos made no contribution to low-temperature physics," the book says, but research on helium-3 originated at Los Alamos, and Matthias' laboratory there played an important role later. And the authors declare that, in contrast to the experimentalists, "it was essentially the great pre-war figures who continued to hold center stage among the theorists." But the immediate post-war generation of theorists was arguably as strong and as numerous as any in history: Feynman, Tsung-Dao Lee, Chen Ning Yang, Julian Schwinger, Murray Gell-Mann and, among the condensed-matter types, David Pines, Philippe Nozieres and Walter Kohn.

There are also errors in personal details that in many cases subtly alter the emphases in the story. Bardeen was not a "student of Slater", he was a junior fellow at Harvard, and no other source has him seriously influenced by John Slater. Ted Geballe was not an "early student" of Matthias; rather, Geballe was his department head and mentor at Bell Labs, and the relationship was two-way. (Geballe was, incidentally, trained at the
University of California, Berkeley, a laboratory that the authors condemn to 'also-ran' status in a parenthetical note.) And the authors ignore Matthias' continuous connection to Bell Labs from before he began studying superconductivity.

The book also provides a false impression of the discovery of the Josephson effect. Brian Josephson's paper predicting tunnelling supercurrents was not ten years after BCS theory - a fact that is given some significance - but five. He was also unable to put his own predictions into experimental practice, and had a public argument as to their validity with Bardeen in the summer of 1962; this was shortly settled by experiments done by John Rowell and me at Bell Labs.

But Josephson makes a late arrival (in chapter 18 of 22) in Cold Wars, which focuses mainly on the period before and immediately after the Second World War. It is almost worth reading the book just for the distressing story of London, whose books laid out the problems that the post-war generations were to solve but who died just before the solutions were to become clear. There is also an excellent view of the prickly personality of Landau and of the terrible dangers that threatened him, Kapitsa and Shubnikov as they laboured well in advance of their Western colleagues from 1935 to 1955.

In describing the controversies about the theory of liquid helium's superfluid phase, the authors include the contributions of Feynman but not the penetrating insights from the early 1950s of Lars Onsager, which in my opinion are of equal status. The story of Bardeen's "relentless pursuit" of the solution to superconductivity is well told, 
as is the description of the nature of the BCS theory (with a debt here to Victor Weisskopf, whose explanation is quoted).

The story thereafter becomes sketchy indeed, and misses many vital points. I might suggest that the authors' relative unfamiliarity with the anglophone world, and their weakness in theory, begin here to warp the coverage. There is emphasis on Pierre-Gilles de Gennes' group in France, with its remarkable collective ethos and a significant number of detailed applications of the BCS ideas to its credit, but does this work stand out so much relative to many things that at the time seemed more important? And I cannot let pass the authors' failure to note that although Alex Müller's great discovery of high-temperature superconductivity in the cuprates was unquestionably motivated by bipolaron theory (not an original concept of Benoy Chakraverty, by the way), that theory is nonetheless generally thought to be wrong. This is far from the first time since Christopher Columbus that a wrong concept motivated a great discovery.

In the discussion of the state of theory in this field, my words in a 2001 article for a Nobel symposium are quoted out of context, misreading or misunderstanding the message that the article was meant to convey, namely, that the source of high-temperature superconductivity is not a mystery, and that theory has not been pointless and futile. The reasons why the misleading popular impression of chaos and controversy in this field is so hard to dispel is not explored here, which is a pity. Still, in these final chapters the authors make some telling points about the overselling of the hopes for practical applications that characterized this period. But this critical observation is never balanced against the value of the MRI industry and other applications of superconductivity - by no means a unique story in the history of tensions between research and technology, where hitting the jackpot is a rarity but adds enormous value when it happens.

This year's Nobels bring out both the strengths and the weaknesses of the book. On the one hand, it is an excellent source for the background of the physics prizes to Vitaly Ginzburg and Alexei Abrikosov. But the intricate history of helium-3, now the source of yet a second physics Nobel, to Tony Leggett, is barely mentioned. I have already noted the absence of MRI, the subject of the physiology prize, from their horizon.

The intriguing piece of scientific history in Cold Wars has not been as well presented elsewhere, and the book is worth the attention of layman as well as scientist. But caveat emptor: the real inside story is not here if you're interested in what actually happened or in just who did what. But I found it refreshing to find judgements as to the broad trends of socio-scientific history, even if some of these were off by a little or, occasion- ally, a lot. Far too often the history of science confines itself to bare facts - when it pays attention to them at all.

Philip W. Anderson is in the Department of Physics, Princeton University, Princeton, New Jersey 08544-0708, USA.

\section{$\cdots \cdots \cdots \cdots \cdots \cdots \cdots \cdots$ The rise and fall of populations}

Complex Population Dynamics: A Theoretical/Empirical Synthesis by Peter Turchin

Princeton University Press: 2003. 456 pp.

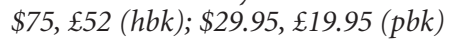

\section{Nils Chr. Stenseth}

People have been fascinated and puzzled for centuries by the profound variations from one year to the next in the abundance of lemmings and populations of hares and lynxes. The archbishop of Uppsala, for example, wrote about the phenomenon as long ago as the fifteenth century. And hunters and other rural people such as the Sami of northern Scandinavia have their own theories to explain the burgeoning populations of lemmings, for instance, in some years. But it was the Oxford zoologist Charles Elton who built the scientific platform for the modern study of population cycles with a 1924 publication in the British Journal of Experimental Biology. That paper and his 1942 book Voles, Mice and Lemmings have been key references ever since.

Much of this work has focused on vertebrates of the north, but similar phenomena have been observed for species in other regions, such as the larch budmoth found in the Alps. The scientific literature on population cycles is vast and has to some extent been characterized by heated debates. So a book that aims to synthesize this rather chaotic field and make it more accessible to outsiders is to be welcomed.

Complex Population Dynamics, written by Russian-born ecologist Peter Turchin, is split into three parts: theory, data and finally a series of six case studies. The theory part provides an excellent synthesis of the work by Turchin and colleagues, but specialists

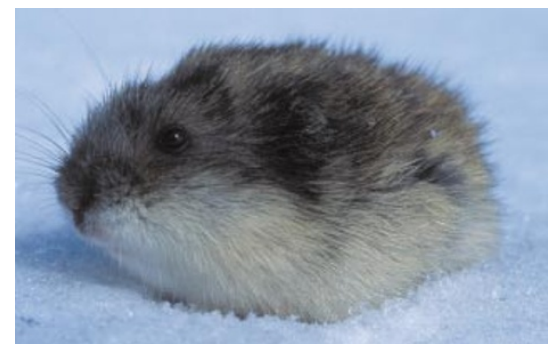

Now you see it... Many suggestions have been made to explain the lemming's disappearing act. on population cycles may find that it does not cover the literature as fully as they might like in a book with ambitions of providing a synthesis of the field. I preferred the second part of the book, which covers both phenomenological (time series-based) and mechanistic modelling — the latter more fully than the former.

The section on case examples is good for the systems that Turchin has worked on himself, but is rather shallow for some of the other systems described, a good exception being the chapter on grouse. However, I think that this book contributes profoundly to the literature, in particular with its emphasis on integrating statistical analysis, theoretical modelling and experiments, rather than relying solely on experimental work. I fully agree with Turchin's conclusion that ecological investigations of population cycles and similar phenomena should start with statistical data analysis, aimed at describing the patterns to be explained, and end with experimental work to discriminate between alternative mechanistic explanations. In this respect the book may have a huge impact on the field, not necessarily because everybody agrees with Turchin's conclusions, but because he provides examples of what a research programme ought to look like.

Turchin's book covers many of the same elements as Population Cycles (Oxford University Press), which was published last year. Edited by Alan Berryman, who contributed an opening chapter and a postscript, Population Cycles comprises seven chapters written by specialists in the field, each considering an example of a population cycle. These chapters, although somewhat variable both in form and quality, display great enthusiasm in attempting to understand why some species and populations exhibit extensive population cycles whereas others do not. A concluding chapter is written by ecologists Xavier Lambin, Charley Krebs, Robert Moss and Nigel Yoccoz, who favour the experimental approach over statistical data analysis. Together with the postscript by Berryman, this provides a good balance regarding methodological approaches - a balance that I am convinced is needed if we are to find the solution to the cycle puzzle.

Both of these books show that the study of population cycles is a stimulating field, with much data and several plausible hypotheses needing to be tested by specifically designed and well-planned experiments. Although both books will provide active scientists in the field with much to think about — and to disagree with - I would not recommend either of them as textbooks, as they both seem too biased or narrow to serve a general educational purpose.

They both express rather similar perspectives, for instance in emphasizing feedback interactions between different trophic levels (between plant and herbivore, predator and 